\title{
Burn-off dominated uranium and asymmetric copper-gold operation in RHIC
}

\author{
Y. Luo, W. Fischer, M. Blaskiewicz, J. M. Brennan, N. Kling, K. Mernick, and T. Roser \\ Brookhaven National Laboratory, Upton, New York 11973, USA
}

(Received 3 December 2013; published 18 August 2014)

\begin{abstract}
In 2012 the Relativistic Heavy Ion Collider collided uranium-uranium ions with a particle energy of $96.4 \mathrm{GeV} /$ nucleon and copper-gold $(\mathrm{Cu}-\mathrm{Au})$ ions at $100 \mathrm{GeV} /$ nucleon for the first time. 3-dimensional stochastic cooling became operational for the first time and greatly enhanced the luminosity. Together with a new lattice configuration, we achieved a burn-off dominated uranium beam lifetime at physics stores. In the asymmetric $\mathrm{Cu}-\mathrm{Au}$ collision, we observed an increased $\mathrm{Cu}$ beam loss stemming from different intrabeam-scattering and cooling rates between the $\mathrm{Au}$ and $\mathrm{Cu}$ ion bunches. By intentionally slowing down the cooling rate for the Au beam at the beginning of store, we reduced the $\mathrm{Cu}$ beam loss and maximized the integrated luminosity.
\end{abstract}

DOI: 10.1103/PhysRevSTAB.17.081003

PACS numbers: 29.27.Bd

\section{INTRODUCTION}

The Relativistic Heavy Ion Collider (RHIC) at Brookhaven National Laboratory consists of two superconducting rings. They intersect horizontally at 6 symmetric interaction points (IPs) along a circumference of $3.8 \mathrm{~km}$. Currently the two beams collide at IP6 (STAR detector) and IP8 (PHENIX detector). RHIC is capable of colliding heavy ions and polarized protons. Before 2012, RHIC had collided gold-gold (Au-Au), deuteron-gold (d-Au), copper-copper $(\mathrm{Cu}-\mathrm{Cu})$ ions, and polarized protons (p-p) at different energies [1,2].

In 2012 we collided uranium-uranium (U-U) ions with a particle energy of $96.4 \mathrm{GeV} /$ nucleon and copper-gold $(\mathrm{Cu}-\mathrm{Au})$ ions at $100 \mathrm{GeV} /$ nucleon for the first time. The first high energy U-U collisions in RHIC were enabled by the versatile new Electron-Beam Ion Source (EBIS) [3-5]. EBIS can produce beams of essentially any ion species and can switch rapidly between two different species. In the previous RHIC ion runs, ions were provided by the Tandem preinjector [6].

Intra-beam scattering (IBS) [7-11] is the main limiting factor to the luminosity in the RHIC heavy ion runs. IBS increases the bunch length and blows up transverse beam sizes, and therefore reduces the beam and luminosity lifetimes [12-15]. To counteract IBS, stochastic cooling had been developed and implemented in the past few years [16-20]. In 2012, by adding the horizontal plane, 3-dimensional (3-d) stochastic cooling became operational for the first time in RHIC.

\footnotetext{
yluo@bnl.gov
}

Published by the American Physical Society under the terms of the Creative Commons Attribution 3.0 License. Further distribution of this work must maintain attribution to the author $(s)$ and the published article's title, journal citation, and DOI.
The luminosity was greatly enhanced with 3-d stochastic cooling [21-25]. For an example, in the 2012 U-U operation, the peak luminosity was 3 times the initial one at the beginning of store, which was observed for the first time in a collider. Together with a new lattice configuration, the particle loss was nearly entirely caused by the luminosity burn-off, which was also observed for the first time in a collider.

In the following, we first briefly review IBS, stochastic cooling, and lattice and beam parameters in the RHIC ion runs. Then we analyze the mechanisms for the nonluminous beam loss in the previous $\mathrm{Au}-\mathrm{Au}$ runs and conclude that it is mainly due to a limited off-momentum dynamic aperture. In 2012 we adopted a new lattice configuration which provides a large off-momentum dynamic aperture and achieved a burn-off dominated $\mathrm{U}$ beam lifetime at physics stores. Next we analyze the causes for a large $\mathrm{Cu}$ beam loss observed at the beginning of store in the asymmetric $\mathrm{Cu}-\mathrm{Au}$ operation and conclude that it is due to beam-beam interaction with different beam sizes at the collisional points stemming from different IBS and cooling rates between the $\mathrm{Au}$ and $\mathrm{Cu}$ ion beams. By intentionally slowing down the cooling rate for the Au beam, we reduced the $\mathrm{Cu}$ beam loss and maximized the store length and integrated luminosity.

\section{RHIC HEAVY ION RUNS}

\section{A. IBS}

Coulomb scattering between charged particles in a stored beam results in an exchange of energy between different degrees of freedom. Two scattering regimes are considered: Touschek effect is large-angle scatterings which transfers a small transverse momentum into a large longitudinal momentum and causes particle loss out of RF buckets, IBS is multiple small-angle scatterings which leads to a 
diffusion process and causes particle distribution changes in all three dimensions.

For a relativistic circular accelerator like RHIC, the longitudinal temperature is much smaller than the transverse one in the beam frame. If the vertical dispersion is neglected, the emittance growth rates due to IBS can be calculated with [7]

$\frac{d}{d t}\left(\begin{array}{c}\epsilon_{x} \\ \epsilon_{y} \\ \sigma_{p}^{2}\end{array}\right)=\frac{N_{i} r_{i}^{2} c}{4 \sqrt{2} \beta^{3} \gamma^{3} \sigma_{z}}\left\langle\frac{L_{c}}{\sigma_{x} \sigma_{y} \theta_{\perp}}\left(\begin{array}{c}\Psi_{x}\left(\theta_{x}, \theta_{y}\right) \\ \Psi_{y}\left(\theta_{x}, \theta_{y}\right) \\ \Psi\left(0, \theta_{x}, \theta_{y}\right)\end{array}\right)\right\rangle_{s}$,

where $\epsilon_{x, y}$ are the unnormalized rms transverse emittances, $\sigma_{x, y, z}$ the transverse and longitudinal $\mathrm{rms}$ bunch sizes, $\sigma_{p}$ the rms relative momentum spread. $N_{i}$ is the particle population per bunch or bunch intensity, $r_{i}$ the classic radius of ions, $\gamma$ relativistic factor, $c$ speed of light, and $\beta c$ the particle velocity. $L_{c}$ is Coulomb logarithm, whose average along the ring is 21.8 for the RHIC ion runs.

In Eq. (1), we used following definitions:

$$
\begin{gathered}
\theta_{\perp}=\sqrt{\theta_{x}^{2}+\theta_{y}^{2}}, \\
\theta_{x}^{2}=\frac{\epsilon_{x}}{\beta_{x}}\left(1+\frac{\sigma_{p}^{2}\left(\Phi_{x} \beta_{x}\right)^{2}}{\sigma_{x}^{2}}\right), \\
\theta_{y}^{2}=\frac{\epsilon_{y}}{\beta_{y}}, \\
\Psi_{x}\left(\theta_{x}, \theta_{y}\right)=\Psi\left(0, \theta_{x}, \theta_{y}\right) A_{x}+\Psi\left(\theta_{x}, \theta_{y}, 0\right) \frac{\beta_{x}}{\gamma^{2}}, \\
\Psi_{y}\left(\theta_{x}, \theta_{y}\right)=\Psi\left(\theta_{x}, \theta_{y}, 0\right) \frac{\beta_{y}}{\gamma^{2}}, \\
\sqrt{2} r \\
\hline \pi, y, z)=\frac{y^{2} R_{D}\left(z^{2}, x^{2}, y^{2}\right)}{\left.+z^{2} R_{D}\left(x^{2}, y^{2}, z^{2}\right)-2 x^{2} R_{D}\left(y^{2}, z^{2}, x^{2}\right)\right],}
\end{gathered}
$$

where $A_{x}=\left(D_{x}^{2}+\left(\beta_{x} \Phi_{x}\right)^{2}\right) / \beta_{x}, \Phi_{x}=D_{x}^{\prime}+\left(\alpha_{x} D_{x}\right) / \beta_{x}$, $\beta_{x, y}$ and $\alpha_{x, y}$ Twiss parameters, $D_{x}$ and $D_{x}^{\prime}$ the horizontal dispersion and its derivative. $R_{D}(x, y, z)$ the symmetric elliptic integral of the second kind, $r=\sqrt{x^{2}+y^{2}+z^{2}}$, $x, y, z \geq 0$.

For an ultrarelativistic machine, considering that $\beta_{x, y} / \gamma^{2} \ll 1$, the vertical emittance growth directly from IBS can be neglected. However, the vertical emittance growth can be coupled from the horizontal plane through betatron coupling. In the RHIC operation, we observed similar horizontal and vertical emittance growth rates. For the $100 \mathrm{GeV} \mathrm{Au}-\mathrm{Au}_{2}$ operation, the longitudinal growth time $\tau_{\|}$, with $\tau_{\|}^{-1}=\frac{1}{\sigma_{p}^{2}} \frac{d \sigma_{p}^{2}}{d t}$, is about 0.3 hour at the beginning of store. The transverse emittance growth times $\tau_{x, y}$, with $\tau_{x, y}^{-1}=\frac{1}{\epsilon_{x, y}} \frac{d \epsilon_{x, y}}{d t}$, are about 0.8 hours with full coupling.

\section{B. Stochastic cooling}

To counteract IBS effects, we have been implementing stochastic cooling in the RHIC rings in the past few years. Stochastic cooling was invented by Simon van der Meer [17] and was demonstrated at the CERN ISR and ICE (Initial Cooling Experiment). A complete theory of cooling unbunched beams was developed and applied at CERN and Fermilab. Bunched beam cooling was demonstrated in ICE and has been observed in several rings designed for coasting beam cooling.

Operational longitudinal cooling of high energy bunched ion beam was first achieved in the 2007 RHIC $100 \mathrm{GeV}$ $\mathrm{Au}-\mathrm{Au}$ run. In 2011, the vertical cooling was implemented. Through betatron coupling, both transverse emittances were cooled. In 2012, by adding the horizontal plane, full 3-dimensional stochastic cooling became operational for the first time in RHIC [18-20].

A simple but useful estimate of stochastic cooling rate is [16]

$$
\frac{1}{\epsilon} \frac{d \epsilon}{d t}=-\frac{W}{N_{i}}\left[2 g-g^{2}(M+U)\right],
$$

where $\epsilon$ is the transverse beam emittances or $\sigma_{p}^{2}, W$ the system's band width, and $g$ the cooling gain. $M$ and $U$ are the mixing factor and the ratio of electronic noise power to the average Schottky power density. From Eq. (8), the stochastic cooling rate is inversely proportional to the bunch intensity $N_{i}$.

The ions are injected into RHIC at $10 \mathrm{GeV} /$ nucleon and are accelerated to the top energy of about $100 \mathrm{GeV} /$ nucleon with $28 \mathrm{MHz}$ RF cavities and harmonic number 360 . We fill 111 bunches, one bunch every $3 \mathrm{RF}$ buckets, in each ring and leave a gap for the beam abort. After reaching the top energy, to generate short bunches, we carry out RF rebucketing from the $28 \mathrm{MHz}$ RF system to a $197 \mathrm{MHz}$ RF system and then bring the two beams into collision. At physics stores, $300 \mathrm{kV} 28 \mathrm{MHz}$ and $3 \mathrm{MV}$ RF voltages are used.

\section{IBS-suppression lattice}

Before the transverse stochastic cooling became operational in RHIC, we explored a way to reduce the transverse IBS growth rates by reducing $A_{x}$ in Eq. (5) [14]. We intentionally increased the phase advances of FODO cells and reduced the horizontal dispersion $D_{x}$ in the arcs. These IBS-suppression lattices have integer tunes $(31,32)$, which are 3 units higher than the original RHIC design integer tunes $(28,29)$. For both lattices, the fractional tunes are $(0.23,0.22)$. We refer to the lattices with the original design integer tunes as the "standard" lattices. 
TABLE I. Optics parameters of the IBS-suppression and the standard lattices for the RHIC ion runs.

\begin{tabular}{|c|c|c|}
\hline Parameter & $\begin{array}{l}\text { IBS-suppression } \\
\text { Lattices }\end{array}$ & Standard Lattices \\
\hline \multicolumn{3}{|l|}{ Both rings: } \\
\hline$\beta^{*} \mathrm{~s}$ at IP6 and IP8 & \multicolumn{2}{|c|}{$0.7 \mathrm{~m}$} \\
\hline$\left(Q_{x}, Q_{y}\right)$ & $(31.23,32.22)$ & $(28.23,29.22)$ \\
\hline$\Delta \Phi_{\mathrm{FODO}}$ & $\sim 95^{\circ}$ & $\sim 84^{\circ}$ \\
\hline$\left\langle\beta_{x}^{1 / 2}\right\rangle$ & 6.0 & 6.7 \\
\hline$\left\langle A_{x}\right\rangle$ & $1.73 \mathrm{~m}$ & $2.42 \mathrm{~m}$ \\
\hline$\alpha_{p}$ & 0.00144 & 0.00184 \\
\hline $\begin{array}{l}\text { Blue ring: } \\
\text { uncorrected } \xi_{x, y}^{(1)}\end{array}$ & $(-102,-98)$ & $(-91,-90)$ \\
\hline $\begin{array}{l}\text { corrected } \xi_{x, y}^{(1)} \\
\xi_{x, y}^{(2)} \text { with corrected } \xi_{x, y}^{(1)}\end{array}$ & $\begin{array}{r}(1, \\
(-4800,-900)\end{array}$ & $(2500,1200)$ \\
\hline Yellow ring: & & \\
\hline uncorrected $\xi_{x, y}^{(1)}$ & $(-101,-99)$ & $(-92,-90)$ \\
\hline corrected $\xi_{x, y}^{(1)}$ & \multicolumn{2}{|c|}{$(1,1)$} \\
\hline$\xi_{x, y}^{(2)}$ with corrected $\xi_{x, y}^{(1)}$ & $(3900,1900)$ & $(-1300,1000)$ \\
\hline
\end{tabular}

Table I shows the lattice parameters based on off-line optics models for both lattices. $\Delta \Phi_{\mathrm{FODO}}$ is the phase advance per FODO cell, $\alpha_{p}$ the momentum compaction factor. $\xi_{x, y}^{(1)}$ and $\xi_{x, y}^{(2)}$ are the first and second order chromaticities. For comparison, $\beta^{*} \mathrm{~s}$ at IP6 and IP8 are set to $0.7 \mathrm{~m}$ for both lattices. The IBS-suppression lattice was experimentally tested in the 2007, and used in the Yellow ring with $\mathrm{Au}$ ion beam in the $2008 \mathrm{~d}-\mathrm{Au}$ run, and in both rings in the 2010 and $2011 \mathrm{Au}-\mathrm{Au}$ runs. Both numeric simulations and beam experiments showed that the IBS-suppression lattices reduced the transverse emittance growth rate by $30 \%[14,15]$.

In the $2011100 \mathrm{GeV} \mathrm{Au-Au}$ run, with the IBSsuppression lattices, two dedicated fills (one after another) were used for comparison without cooling and with longitudinal and vertical cooling. Figure 1 shows the averaged bunch intensities, normalized transverse rms emittances, rms bunch lengths, and luminosities. The bunch lengths are for the central bunch. Satellite bunches emerged on both sides of the central one throughout the store. From Fig. 1, longitudinal and vertical cooling reduced bunch length and transverse emittance, and improved the beam and luminosity lifetimes. The integrated luminosity per store was almost doubled with longitudinal and vertical cooling.

\section{Beam parameters in 2012 run}

Table II lists parameters of ions used in the 2012 RHIC ion run. The typical $\mathrm{U}$ ion bunch intensity in RHIC was $0.3 \times 10^{9}$, which was half of its projection. The lower $\mathrm{U}$ ion bunch intensity was due to the lower intensities from the preinjector and the lower than expected foil stripping and ion transfer efficiencies. To increase the bunch intensity, double bunch merging was implemented in both the Booster and AGS. Double bunch merging became
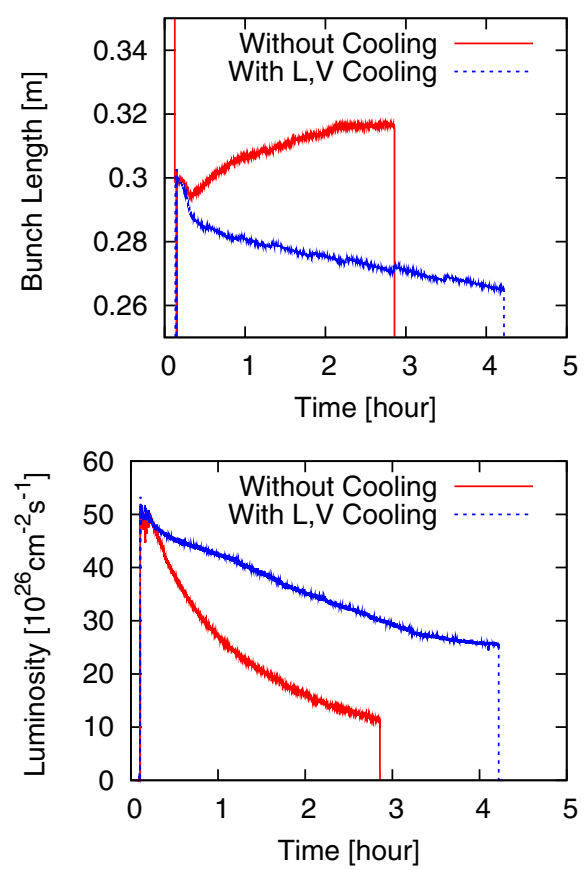

FIG. 1. Comparison of two fills in $2011100 \mathrm{GeV} \mathrm{Au-Au} \mathrm{run,} \mathrm{one} \mathrm{without} \mathrm{cooling} \mathrm{and} \mathrm{one} \mathrm{with} \mathrm{longitudinal} \mathrm{and} \mathrm{vertical} \mathrm{cooling.}$ Top-left: bunch intensities. Top-right: rms bunch lengths of central bunch. Bottom-left: transverse emittances. Bottom-right: luminosities at IP8 from Zero Degree Calorimeters (ZDCs). This comparison was made with IBS-suppression lattices. 
TABLE II. Ion and beam parameters in the 2012 ion run.

\begin{tabular}{lccccc}
\hline \hline & & & \multicolumn{3}{c}{ Ion Species } \\
\cline { 4 - 6 } Parameter & Symbol & Unit & $\mathrm{U}$ & $\mathrm{Cu}$ & $\mathrm{Au}$ \\
\hline Mass number & $A$ & $\ldots$ & 238 & 63 & 197 \\
Charge state & $Z$ & $\ldots$ & 92 & 29 & 79 \\
Energy & $E$ & $\mathrm{GeV} /$ nucleon & 96.4 & 100 & 100 \\
Bunch Intensity & $N_{i}$ & $10^{9}$ & 0.3 & 4.0 & 1.3 \\
Emittances & $\epsilon_{n}$ & $\mu \mathrm{m}$ & 1.5 & 3.5 & 2.5 \\
\hline \hline
\end{tabular}

operational two days before the end of the U-U run and was used in the whole following $\mathrm{Cu}-\mathrm{Au}$ run.

Through the course of the $\mathrm{Cu}-\mathrm{Au}$ run, the $\mathrm{Cu}$ bunch intensity was doubled and the Au bunch intensity increased by $50 \%$. The $\mathrm{Cu}$ and $\mathrm{Au}$ bunch intensities reached $4.0 \times 10^{9}$ and $1.3 \times 10^{9}$ at the end of the $\mathrm{Cu}-\mathrm{Au}$ run. With 3-d cooling at store, we tolerated emittance blow-up during acceleration until the bunch intensity limit at transition was reached [26-29].

In the following, we only use normalized transverse emittance $\epsilon_{n}, \epsilon_{n}=\gamma \epsilon$. The typical initial normalized transverse rms emittance for the U beam was $1.5 \mu \mathrm{m}$. Both the longitudinal and transverse emittances for $\mathrm{Cu}$ and $\mathrm{Au}$ beam increased with the bunch intensities due to double bunch merges. For an example, with a bunch intensity $4.0 \times 10^{9}$, the $\mathrm{Cu}$ beam's emittance could reach $3.5 \mu \mathrm{m}$.

We adopted the standard lattices with $\beta^{*}=0.7 \mathrm{~m}$ at IP6 and IP8 for both the U-U and $\mathrm{Cu}-\mathrm{Au}$ operation. The reason to switch back to the standard lattices will be explained in the next session. Standard lattices with $\beta^{*}=0.7 \mathrm{~m}$ were not operated before 2012. The $\beta^{*}$ s at the noncollisional IPs were $5 \mathrm{~m}$. The operational fractional tunes are $(0.235,0.23)$ for the Blue ring and $(0.229,0.226)$ for the Yellow ring.

\section{BURN-OFF DOMINATED BEAM LIFETIME IN THE U-U OPERATION}

\section{A. Nonluminous particle loss}

Nonluminous particle loss had been always observed at physics stores in the RHIC ion runs before 2012, especially with the IBS-suppression lattices in 2010 and 2011 [30,31]. We define the instantaneous total beam loss rate $\tau_{\text {tot }}^{-1}(t)$ as

$$
\tau_{\mathrm{tot}}^{-1}(t)=-\frac{1}{N_{\mathrm{tot}, i}(t)} \frac{d N_{\mathrm{tot}, i}(t)}{d t} .
$$

$\tau_{\text {tot }}$ is the beam lifetime in units of hours, $N_{\text {tot, } i}$ the total beam intensity. In this article, the beam loss rate is given in units of \%/hour.

Figure 2 shows the beam loss rate and burn-off contribution in the Yellow ring for the fill with cooling in Fig. 1. The burn-off caused beam loss rate is calculated with

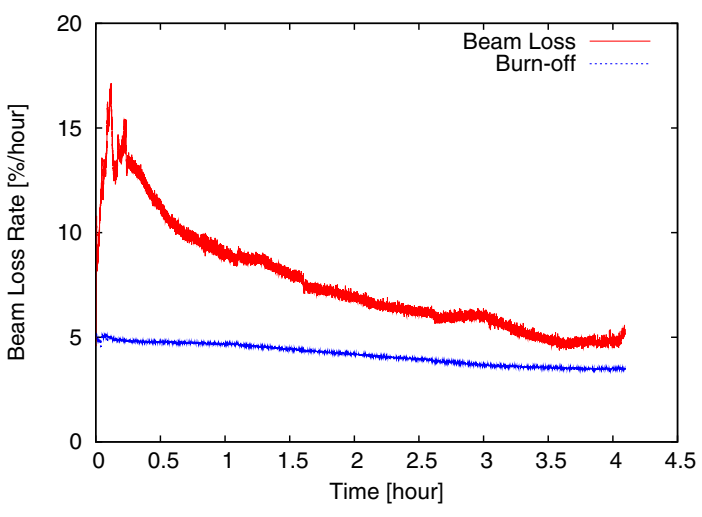

FIG. 2. One example of nonluminous beam loss of the Au beam with longitudinal and vertical cooling in the Yellow ring in the $2011100 \mathrm{GeV}$ Au-Au run.

$$
\tau_{\text {burn-off }}^{-1}(t)=\frac{\left(L_{\mathrm{IP} 6}(t)+L_{\mathrm{IP} 8}(t)\right) \sigma_{\mathrm{coll}}}{N_{\mathrm{tot}, i}(t)}
$$

where $\sigma_{\text {coll }}$ is the total cross section of ion collision, $L_{\mathrm{IP} 6 \text {,IP8 }}$ the instantaneous luminosities from the two detectors at IP6 and IP8. The analytically calculated total cross-section for the $100 \mathrm{GeV} /$ nucleon $\mathrm{Au}-\mathrm{Au}$ ion collision is $218.46 \mathrm{~b}$ [32], which is used for the burn-off calculation in this article.

In Fig. 2, the total beam loss rate exceeded 15\%/hour at the beginning of store and decreased to $5 \% /$ hour at the end of store. The burn-off contributed beam loss rate stayed around 5\%/hour to $4 \% /$ hour throughout the store. The nonluminous beam loss rate reached $10 \%$ /hour at the beginning and about $1 \%$ hour at the end of store. Integrating over the whole store, the nonluminous beam loss accounts for $46 \%$ of the total beam loss.

Nonluminous beam loss reduces beam and luminosity lifetimes and therefore should be minimized. The causes for nonluminous beam loss include: (i) beam-gas interaction, (ii) Touschek effect and IBS, (iii) beam-beam interaction, (iv) dynamic aperture, and so on. The first two candidates are not related to collision. In operation, without RF rebucketing and collision, the beam loss rate at the top energy could reach $0.7 \%$ /hour with a low Au ion bunch intensity $0.3 \times 10^{9}$ and $1.2 \%$ /hour with a normal bunch intensity $1.3 \times 10^{9}$. Therefore, beam-gas interaction, Touschek effect and IBS are not the causes for the large nonluminous beam loss.

In Appendix A, we recalculate the ion beam lifetime and emittance growth due to beam-gas interaction with current RHIC vacuum parameters. The dominant beam-gas interaction leading to ion beam loss is the inelastic nuclear collision between the nuclei of ions and gas atoms. For $100 \mathrm{GeV} /$ nucleon $\mathrm{Au}$ ion beam, the beam loss rate due to inelastic nuclear collision is estimated as $0.6 \%$ /hour. The dominant beam-gas interaction leading to ion beam emittance growth is the multiple elastic Coulomb scatterings 
between the nuclei of ions and gas atoms, which can be neglected compared to the IBS contribution.

Beam-beam interaction generates beam-beam tune spread and nonlinear beam-beam resonance driving terms. For the $100 \mathrm{GeV} /$ nucleon Au-Au collision, with a typical Au ion bunch intensity $1.3 \times 10^{9}$ at the beginning of store, the beam-beam parameter with 2 collisions per turn was 0.005. After the Au beam was cooled down, it only reached 0.006 since the bunch intensity decreased. Experimentally, with collision but without RF rebucketing, the beam loss rate was about $5 \%$ /hour at the beginning of store, which was mainly from the burn-off contribution. Numeric simulation also shows that beam-beam interaction below 0.006 does not reduce the dynamic aperture if the fractional tunes are away from the 9th order resonance at 2/9.

In operation, with RF rebucketing but without collision, we observed a large beam loss which could be more than $10 \%$ /hour. Therefore, the observed large nonluminous beam loss was related to RF rebucketing. RF rebucketing reduces the bunch length but increases the momentum spread. With a typical longitudinal emittance $1.0 \mathrm{eV} \cdot \mathrm{s} /$ nucleon, the relative momentum spread of particles is $0.6 \times 10^{-3}$ before rebucketing. After rebucketing, it increases to $1.7 \times 10^{-3}$. The observed large nonluminous beam loss with RF rebucketing indicates a small off-momentum dynamic aperture.

\section{B. Longitudinal particle motion}

Figure 3 shows the longitudinal phase space with $0.3 \mathrm{MV} 28 \mathrm{MHz}$ and $3 \mathrm{MV} 197 \mathrm{MHz} \mathrm{RF}$ voltages. The relative momentum acceptance is $1.4 \times 10^{-3}$ for the central $197 \mathrm{MHz}$ RF bucket and $1.8 \times 10^{-3}$ for the dual RF system. RHIC longitudinal stochastic cooling is based on the $197 \mathrm{MHz} \mathrm{RF}$ system. Its longitudinal acceptance is $1.8 \times 10^{-3}$. Particles can be cooled in the nearest bucket.

Figure 4 shows the measured longitudinal bunch profiles: (i) without rebucketing, (ii) right after rebucketing, and (iii) with cooling for 2 hours for a same fill in 2011.

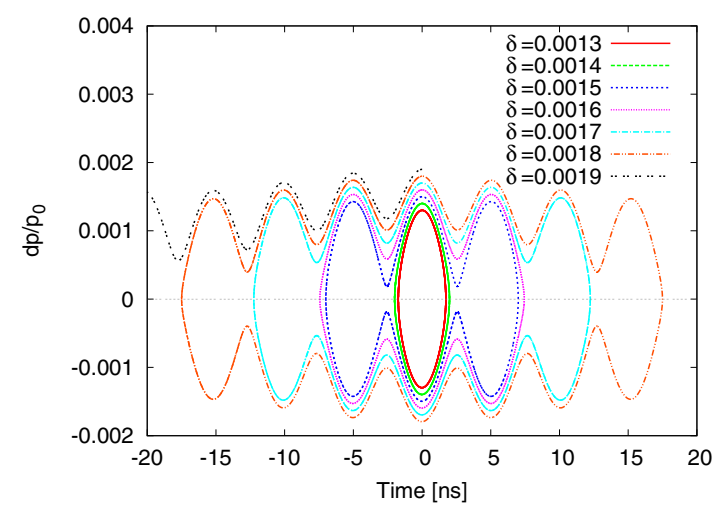

FIG. 3. Longitudinal phase space in the RHIC ion runs. The total voltages for the $28 \mathrm{MHz}$ and $197 \mathrm{MHz}$ RF systems are $0.3 \mathrm{MV}$ and $3 \mathrm{MV}$.

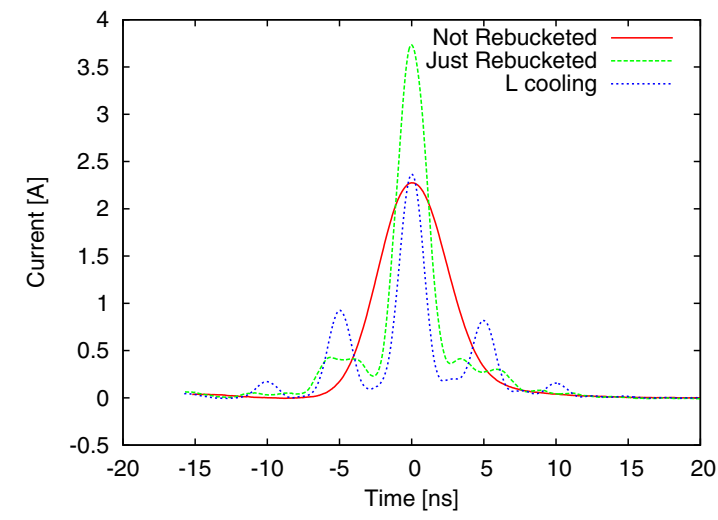

FIG. 4. Measured longitudinal bunch profiles for a same fill: without rebucketing (red), shortly after rebucketing (green), and with longitudinal cooling for 2 hours at store (blue).

The longitudinal and vertical cooling was on from the beginning of store. The longitudinal bunch profiles were measured with the wall current monitors. The vertical axis in Fig. 4 is line current.

After RF rebucketing, not all the particles were captured into the central bucket, as shown by the green curve. Particles out of the central bucket have a relative momentum deviation larger than $1.4 \times 10^{-3}$. If the off-momentum dynamic aperture is not big enough, some of them will get lost in the transverse plane. They actually contributed to the large nonluminous beam loss at the beginning of store, as shown in Fig. 2. Longitudinal cooling could not save the early particle loss due to its finite cooling time.

With longitudinal cooling on, there were still particles leaking out of the central RF bucket, as shown by the blue curve in Fig. 4. These particles also have a relative momentum deviation larger than $1.4 \times 10^{-3}$. Some of them were cooled into the adjacent buckets and formed the satellite bunches. Some of them with a larger offmomentum deviation were lost. We did not observe unbunched beam with longitudinal cooling on. The nonluminous beam loss should take place in the transverse plane due to a limited off-momentum dynamic aperture.

Here we calculate the loss rate of particles which leaked out of the central RF bucket but did not get into the satellite buckets. Figure 5 shows the result, compared with the nonluminous beam loss rate. The same fill as in Fig. 2 was used. The percentage loss rate is with respect to the bunch intensity. The numbers of particles in the central and satellite buckets were calculated with the longitudinal bunch profiles. Burn-off caused particle population changes to the central and satellite bunches are also taken into account. Figure 5 shows a good agreement between the loss rate from particles leaking out of the central bucket but not getting into the satellite buckets and the observed nonluminous particle loss rate.

There are two processes which can move particles out of the central RF bucket: Touschek effect and IBS. 


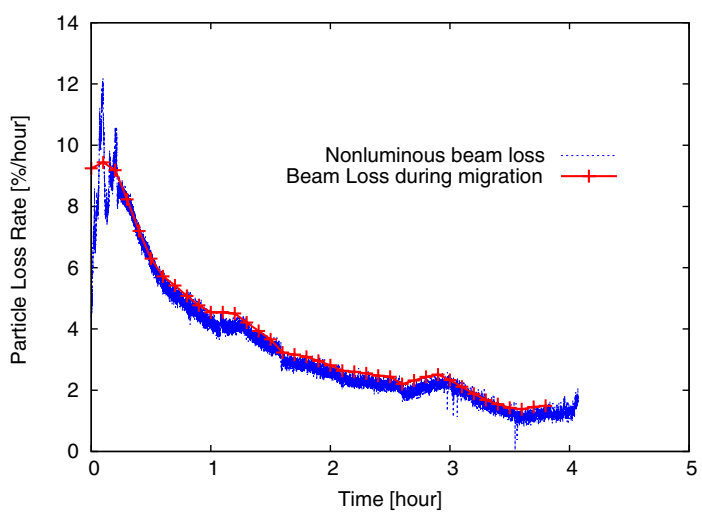

FIG. 5. Loss rate of particles leaking out of the central bucket but not getting into the satellite buckets (red) compared with the nonluminous beam loss rate (blue).

Considering that particles filled up the whole central RF bucket, both processes can be important. In Appendix B, we calculated the contribution from Touschek effect. Assuming a Gaussian distribution, we numerically calculated the particle leakage rate from the central bucket due to Touchek effect to be $3.7 \%$ /hour at the beginning of store. With a total particle leakage rate of $20 \%$ /hour from the central bucket, IBS is the main reason to move the particles out of the central bucket. After 2.5 hours into store when the Au beam was cooled down, the particle leakage rate from the central bucket due to Touschek effect increased to $5.4 \% /$ hour. With a total leakage rate of $6.5 \%$ hour, Touschek effect became the main reason to move particles out of the central bucket.

Numeric simulation with IBS and stochastic cooling has been performed to benchmark the beam sizes and to model the luminosity evolution [33]. We slice each bunch longitudinally and calculate each slice's IBS growth rates using the formulas for coasting beam. The kicks to macroparticles are randomly assigned. Simulation reproduces the observed longitudinal bunch profiles without cooling. Figure 6 shows the bunch profiles with longitudinal cooling from

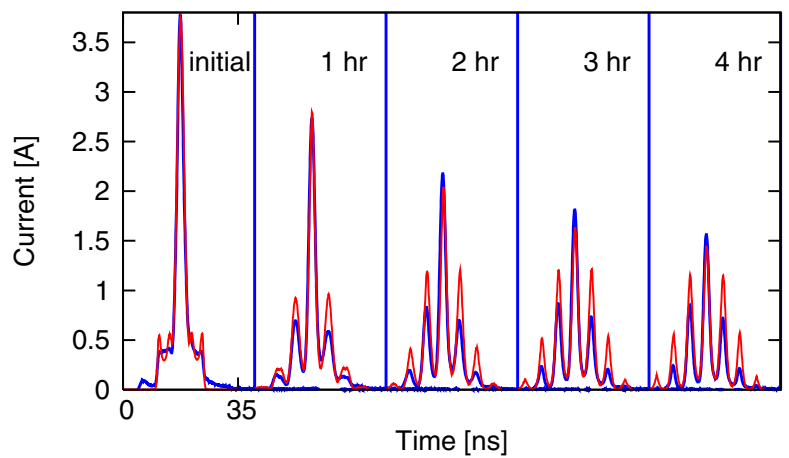

FIG. 6. Comparison of longitudinal bunch profiles between measurement and simulation. There are more particles in satellite buckets from simulation than the measurements. simulation, compared to the measurements. There are less particles in the satellite buckets from the measurements than the simulation, which hints that there are particles leaking out of the central bucket but not getting into the satellite ones. Currently the simulation code does not include off-momentum dynamic aperture and Touschek effect.

\section{Off-momentum dynamic aperture}

Here we calculate the off-momentum dynamic apertures of IBS-suppression lattices and compare them to the standard lattices. In this study, particles are tracked element-by-element up to $10^{6}$ turns [34]. The nonlinear field errors in the superconducting magnets in the interaction regions are included. Before tracking, the fractional tunes are set to $(0.235,0.23)$, and the first order chromaticities are set to $(1,1)$. For each case, we search the dynamic aperture in 10 equally spaced phase angles in the first quadrant of the $\left(x / \sigma_{x}, y / \sigma_{y}\right)$ plane. We only focus on the minimum dynamic apertures for different tracking conditions. The dynamic aperture is given in units of transverse rms beam size. The initial relative momentum deviation $d p / p_{0}$ of particles varies from 0 up to $1.8 \times 10^{-3}$ with a step size of $0.2 \times 10^{-3}$.

Second order chromaticity correction to each lattice is implemented in simulation. Several correction methods and optimization algorithms were used to minimize the second order chromaticities and geometric resonance driving terms [35-40]. For an example, for each arc, we can increase the strength of one focusing or defocusing subfamily and reduce the strength of the other focusing or defocusing subfamily at the same time by the same amount. By doing so, the second order chromaticites can be minimized while the first order chromaticity is kept almost unchanged. Polarity reversals of sextupoles and very large or very small correction strengths are also avoided. With this method and moderate correction strengths, in simulation we could correct the second order chromaticities of the IBS

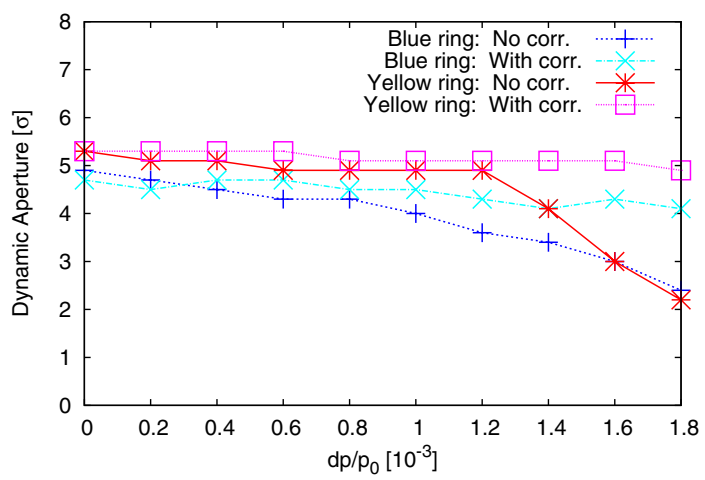

FIG. 7. Calculated off-momentum dynamic apertures for the IBS-suppression lattices without and with second order chromaticity correction. 


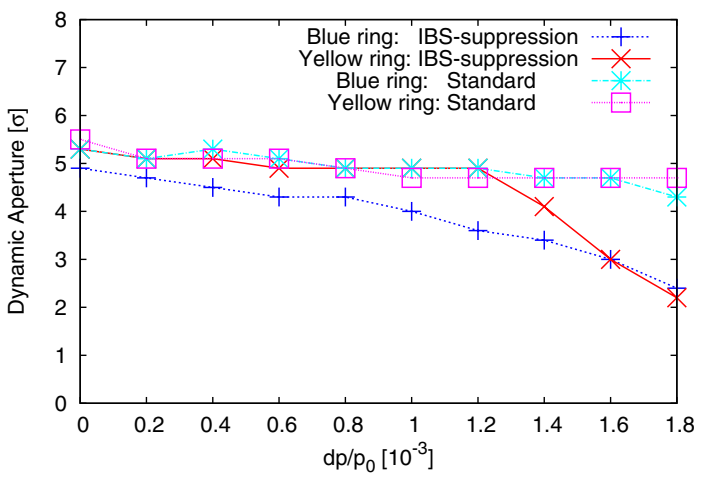

FIG. 8. Calculated off-momentum dynamic apertures for the IBS-suppression lattices and standard lattices. Second order chromaticity corrections are not included.

suppression lattices down below 500 for the Blue ring and down below 1500 for the Yellow ring.

Figure 7 shows the calculated off-momentum dynamic aperture versus the initial relative momentum deviation $d p / p_{0}$ for the IBS-suppression lattices without and with the second order chromaticity correction. Without correction, the off-momentum dynamic apertures are only $2.4 \sigma$ for the Blue ring and $2.2 \sigma$ for the Yellow ring at $d p / p_{0}=1.8 \times 10^{-3}$, which caused a large nonluminous beam loss as discussed above. With second order chromaticity correction, the off-momentum dynamic aperture is increased to $4.1 \sigma$ for the Blue ring and to $4.9 \sigma$ for the Yellow ring. In this simulation, the second order chromaticity correction in the Blue ring puts the strengths of some focusing sextupoles close to zero, which may be a concern for the real operation.

Then we reexamined the off-momentum dynamic aperture for the standard lattices with the same $\beta^{*}=0.7 \mathrm{~m}$. Figure 8 compares the calculated off-momentum dynamic apertures for the IBS-suppression and standard lattices. Second order chromaticity corrections are not included in this comparison. At $d p / p_{0}=1.8 \times 10^{-3}$, the offmomentum dynamic apertures for the standard lattices are $4.7 \sigma$ for the Blue ring and $4.3 \sigma$ for the Yellow ring, respectively, which are more than $2 \sigma$ larger than those from the IBS-suppression lattices. For the Blue ring, the standard lattice even provides a larger off-momentum dynamic aperture than the IBS lattice with second order chromaticity correction.

Further second order chromaticity corrections to the standard lattices had also been examined with multiple sextupole families with different accelerator codes [34,41,42]. In the best case, the off-momentum dynamic aperture could be further improved by $0.5 \sigma$ at $d p / p_{0}=1.8 \times 10^{-3}$. However, the correction strengths from all these codes require polarity reversals of some focusing sextupoles, which is not practical in the real operation. The power supplies of sextupoles in RHIC are all unipolar.

\section{Observations in the $2012 \mathrm{U}-\mathrm{U}$ run}

Based on the nonluminous beam loss observations and off-line off-momentum dynamic aperture calculations,
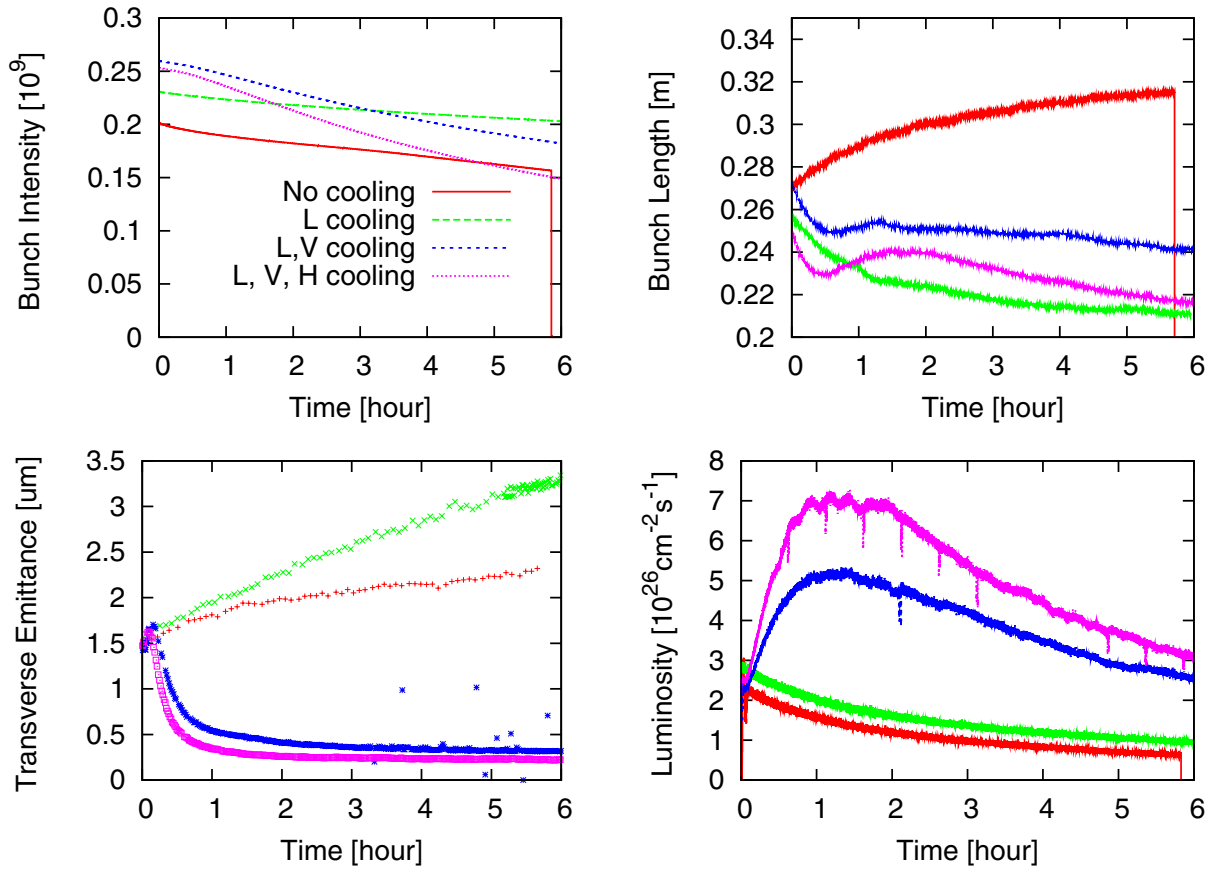

FIG. 9. Comparison of 4 fills without cooling (red), with longitudinal cooling (green), with longitudinal and vertical cooling (blue), and with 3-d cooling (pink) in the 201296.4 GeV U-U run. Top left: averaged bunch intensity. Top right: rms bunch length of the central bunch. Bottom left: rms transverse emittance. Bottom right: luminosity measured at IP8 with ZDCs. 
we decided to adopt the standard lattices with $\beta^{*}=0.7 \mathrm{~m}$ for both $\mathrm{U}-\mathrm{U}$ and $\mathrm{Cu}-\mathrm{Au}$ runs in 2012. Another reason is that with both transverse cooling available, it is not so critical for a lattice to reduce the transverse IBS growth rate rather than to provide a large off-momentum dynamic aperture to reduce the nonluminous beam loss. Two families of sextupoles for linear chromaticity correction was used at the beginning of U-U run. Further chromaticity correction with multiple families was not necessary as for the nonluminous beam loss.

The typical U ion bunch intensity was $0.3 \times 10^{9}$, which is $1 / 4$ of the typical Au bunch intensity $1.3 \times 10^{9}$. As a result, the IBS growth rate was smaller and the cooling time was shorter for the $\mathrm{U}$ beam. The equilibrium between the IBS and cooling could be reached with a smaller transverse emittance. With a smaller transverse emittance, the uranium ions had a better chance to survive in the transverse plane during their migration from the central to the satellite buckets.

Figure 9 shows the typical bunch intensities, rms bunch lengths of the central bunch, rms transverse emittances, and luminosities without cooling and with different combinations of cooling. From Fig. 9, with 3-d cooling, the transverse beam emittance was reduced by a factor of 5 in 1 hour at store, from $1.5 \mu \mathrm{m}$ to $0.3 \mu \mathrm{m}$. The peak luminosity was reached 1 hour into the store and was 3 times the initial one. The total beam-beam parameter increased from 0.001 at the beginning of store to 0.0045 when the beam was cooled. No extra beam loss due to beam-beam interaction was observed.

Figure 10 shows the total beam loss rate and burn-off contribution. With the standard lattice which provides a large off-momentum dynamic aperture, the particle loss was nearly entirely from burn-off. The luminous beam loss rate reached $10 \%$ /hour when the $\mathrm{U}$ beam was deeply cooled. By fitting the beam loss rates against the sum of luminosities at IP6 and IP8 for all qualified stores, we

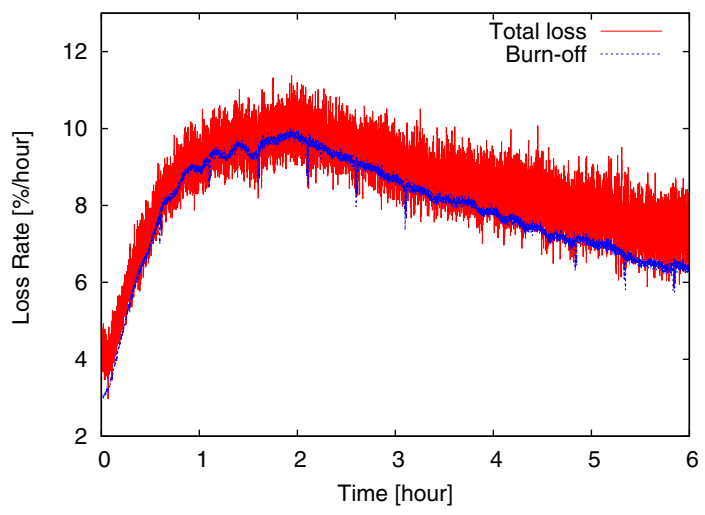

FIG. 10. The beam loss rate and burn-off contribution with 3-d cooling in the 2012 RHIC 96.4 GeV U-U run. Almost all beam loss was from burn-off. experimentally measured the total collision cross section of $100 \mathrm{GeV} /$ nucleon U-U collision, [32]

$$
\sigma_{\text {tot }}^{\text {meas }}=\left(515 \pm 13^{\text {stat }} \pm 22^{\text {sys }}\right) \text { b. }
$$

Compared to the analytically calculated total cross section $487.3 \mathrm{~b}$, we concluded that nearly entire particle loss were from luminosity burn-off. The dominant remaining nonluminous particle loss was from beam-gas interaction.

Figures 11 and 12 show the bunch profile and luminosity evolutions from simulation. In simulation, the initial distribution is assumed as Gaussian, which is slightly different from the real one especially at the bunch tail. Simulation largely reproduces the observed bunch profiles and luminosities throughout the whole store. Comparing to the previous $\mathrm{Au}-\mathrm{Au}$ operation with IBS-suppression lattices, the difference between the simulated and measured bunch profiles was reduced. This means that there was less loss of particles during their migration from the central to satellite buckets. The overshot luminosity from simulation at the

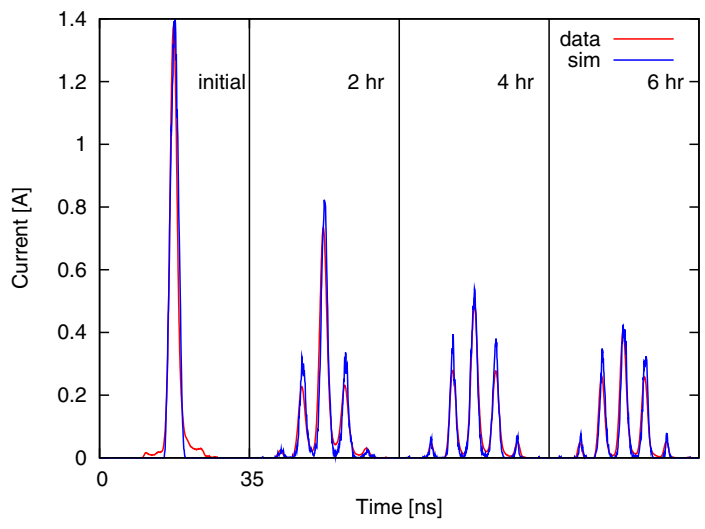

FIG. 11. Comparison of longitudinal bunch profiles from simulation ("sim") and from measurement ("data") for the 2012 RHIC 96.4 GeV U-U run.

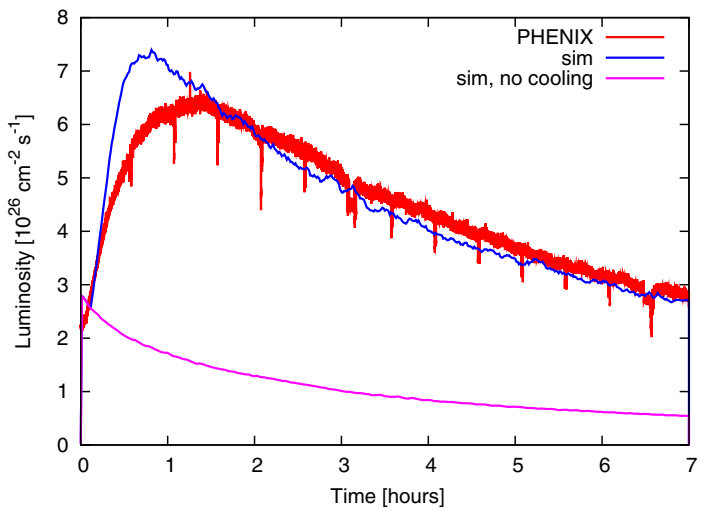

FIG. 12. Comparison of luminosities from simulation ("sim") and from IP8 ZDCs ("PHENIX") for the 2012 RHIC $96.4 \mathrm{GeV}$ U-U run. The simulated luminosity without cooling ("sim, no cooling") is also presented. 
beginning of store is related to the mismatched initial bunch profile.

\section{BEAM-BEAM INTERACTION IN THE ASYMMETRIC CU-AU OPERATION}

\section{A. Observations with full gain of 3-d cooling}

At the beginning of 2012 asymmetric $\mathrm{Cu}$-Au operation, only with longitudinal and vertical cooling, we readily observed that the $\mathrm{Cu}$ beam loss rate was higher than the $\mathrm{Au}$ beam loss rate. The maximum $\mathrm{Cu}$ beam loss rate could reach $10 \%$ /hour. After horizontal cooling was added, the $\mathrm{Cu}$ beam loss rate was even larger and could reach $30 \%$ /hour. During the whole store, the Au beam loss was below $5 \%$ /hour.

The top-left and top-right plots of Fig. 13 show the bunch intensities and beam loss rates for one physics store with 3 -d cooling. The initial bunch intensities were $2.3 \times 10^{9}$ for the $\mathrm{Cu}$ beam and $0.8 \times 10^{9}$ for the $\mathrm{Au}$ beam. The $\mathrm{Cu}$ bunch intensity drop fast between 1 to 4 hours into store and the maximum beam decay reached $30 \% /$ hour. One feature is that the $\mathrm{Cu}$ beam loss was much smaller at the beginning of store and after 4 hours into store.

The burn-off contributions to the beam loss rates of $\mathrm{Cu}$ and $\mathrm{Au}$ beams are calculated based on the analytically calculated values of cross sections for the $100 \mathrm{GeV} /$ nucleon $\mathrm{Cu}-\mathrm{Au}$ collision, $30 \mathrm{~b}$ for the $\mathrm{Cu}$ beam and $36 \mathrm{~b}$ for the Au beam [43]. The burn-off caused beam loss rates were below $2.5 \%$ /hour for both beams during the
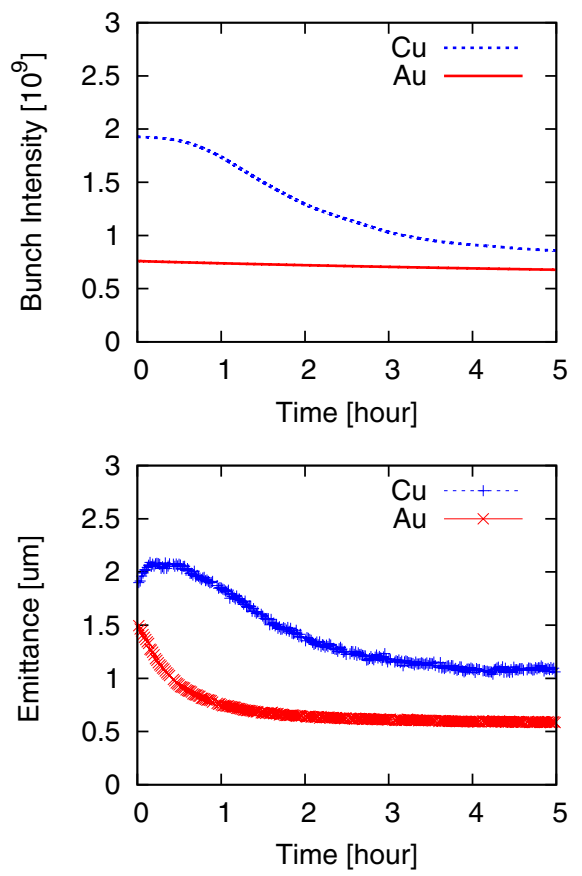

whole store. Burn-off cannot explain the large $\mathrm{Cu}$ beam loss between 1 to 4 hours into store.

The bottom-left plot of Fig. 13 shows the measured transverse rms emittances for both beams. The initial $\mathrm{Cu}$ beam's transverse emittance was 30\% larger than the $\mathrm{Au}$ beam's. The emittance cooling rates were different for both beams. It took about 1 hour to cool down the Au beam's transverse emittance to the equilibrium between IBS and cooling, while the same process took about 3 hours for the $\mathrm{Cu}$ beam. At the equilibrium, the transverse emittance for the $\mathrm{Cu}$ beam was double that for the $\mathrm{Au}$ beam.

\section{B. Different IBS and cooling rates}

First we compare the IBS growth rates and stochastic cooling rates for the $\mathrm{Cu}$ and $\mathrm{Au}$ beams. According to Eq. (1), the IBS growth rate is proportional to $N_{i} r_{i}^{2}$, with $r_{i} \approx Z^{2} r_{p} / A$, for the same lattice and beam sizes. With the typical bunch intensities of $4.0 \times 10^{9}$ for the $\mathrm{Cu}$ beam and $1.3 \times 10^{9}$ for the $\mathrm{Au}$ beam in the $\mathrm{Cu}-\mathrm{Au}$ run, $N_{i, \mathrm{Cu}} \approx 3 N_{i, \mathrm{Au}}$. Then we have

$$
\tau_{\mathrm{IBS}, \mathrm{Cu}}^{-1} \approx \frac{1}{2} \tau_{\mathrm{IBS}, \mathrm{Au}}^{-1}
$$

Based on this, we normally injected the $\mathrm{Cu}$ bunches into RHIC first to reduce the Au beam's emittance growth from IBS at injection.

The stochastic cooling rate is proportional to $1 / N_{i}$, and we have
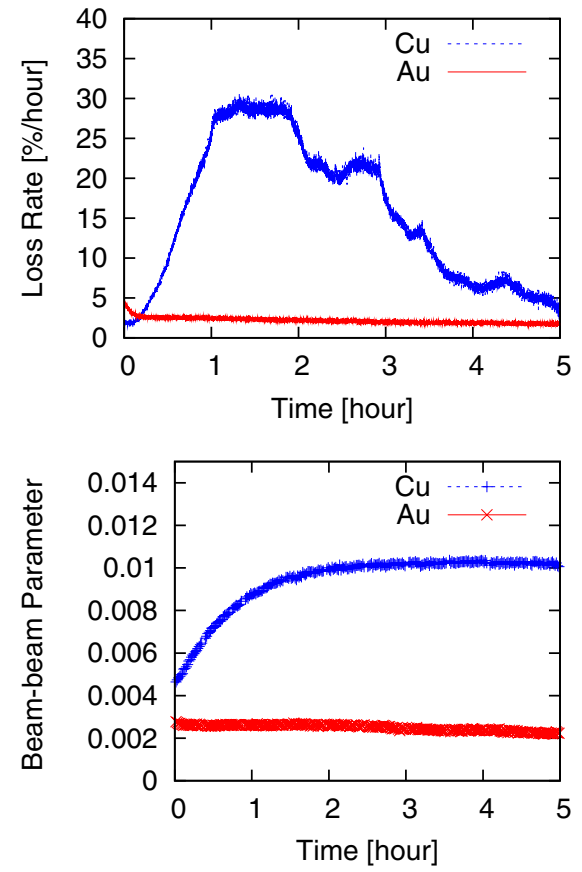

FIG. 13. One fill in the 2012 RHIC $100 \mathrm{GeV}$ asymmetric $\mathrm{Cu}$-Au run with full gains of 3-d cooling. Large beam loss was observed between 1 to 4 hours into store. Top left: bunch intensities. Top right: beam loss rates. Bottom left: normalized transverse emittances. Bottom right: total beam-beam parameters with 2 collisions per turn. 


$$
\tau_{\text {cooling, } \mathrm{Cu}}^{-1} \approx \frac{1}{3} \tau_{\text {cooling,Au }}^{-1}
$$

With different IBS growth and stochastic cooling rates for the $\mathrm{Cu}$ and $\mathrm{Au}$ beams, we observed different $\mathrm{Cu}$ and $\mathrm{Au}$ emittances throughout the store. With the same $\beta^{*} \mathrm{~s}$ at the collisional IPs, different emittances result in different transverse beam sizes at the collisional points.

\section{Unbalanced beam-beam interaction}

For ion collisions, the beam-beam parameter or the linear incoherent beam-beam tune shift with 2 collisions per turn for the weak beam is

$$
\xi_{1}=-\frac{N_{i, 2} r_{p}}{2 \pi \epsilon_{n, 2}} \times \frac{Z_{1} Z_{2}}{A_{1}} .
$$

Here the subscript " 1 " represents the weak beam and " 2 " the strong beam. $Z$ and $A$ are the charge state and mass number of ions, $r_{p}$ the classical radius of proton. For our study of beam-beam effects on the $\mathrm{Cu}$ ions, the $\mathrm{Cu}$ beam is treated as the weak beam even though its bunch intensity is higher, while the Au beam is the strong beam.

The bottom-right plot of Fig. 13 shows the calculated beam-beam parameters for the same store. As the Au beam was continuously cooled down in the first hour of store, the beam-beam parameter for the $\mathrm{Cu}$ beam continuously increased, from 0.004 up to 0.01 . The beam-beam parameter for the $\mathrm{Au}$ beam remained at 0.003 throughout the whole store.

In the following, we perform numeric simulations to calculate the beam-beam effect on the $\mathrm{Cu}$ beam's dynamic aperture in the asymmetric $\mathrm{Cu}-\mathrm{Au}$ collision. In this study, we still track particles element-by-element up to $10^{6}$ turns [34]. The $\mathrm{Cu}$ and $\mathrm{Au}$ bunches collide at IP6 and IP8. 6-D symplectic beam-beam interaction based on sychro-beam mapping $[44,45]$ is adopted. The initial momentum deviation of the test $\mathrm{Cu}$ particles is set to $1.5 \times 10^{-3}$. The total voltages for the $28 \mathrm{MHz}$ and $197 \mathrm{MHz}$ RF cavities are $0.3 \mathrm{MV}$ and $3 \mathrm{MV}$, respectively.

First, we take the bunch intensities and beam emittances from the above store as shown in Fig. 13. Figure 14 shows the calculated $\mathrm{Cu}$ beam's dynamic aperture in the first 4.5 hours into the store. The dynamic apertures are given in units of the $\mathrm{Cu}$ beam's rms transverse beam size. Since the $\mathrm{Cu}$ beam's emittance was changing during this period, the actual value of $1 \sigma$ in units of mm also varied with time.

From Fig. 14, when the two beams were brought into collision, the calculated dynamic aperture for the $\mathrm{Cu}$ ions is $5.5 \sigma$. During the first hour at store, with the transverse emittance of the Au beam continuously cooled down, the dynamic aperture of the $\mathrm{Cu}$ beam was reduced. When the $\mathrm{Au}$ beam was fully cooled, the dynamic aperture of the $\mathrm{Cu}$ beam reached its lowest point at $4.7 \sigma$. With the transverse emittance of $\mathrm{Cu}$ beam continuously cooled down, the

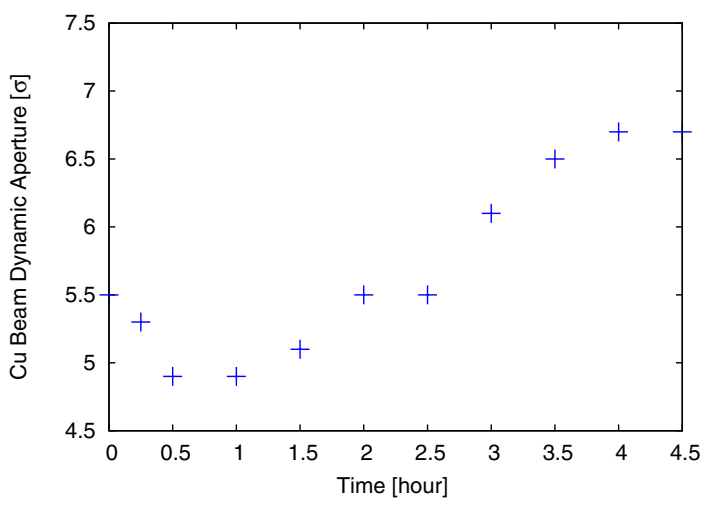

FIG. 14. Calculated dynamic aperture for the $\mathrm{Cu}$ beam with full gains of 3-d stochastic cooling. Bunch intensities and emittances were taken from the fill shown in Fig. 13. The initial particle's relative momentum deviation is $1.5 \times 10^{-5}$.

dynamic aperture for the $\mathrm{Cu}$ beam began to recover. At 2.5 hours into the store, the calculated dynamic aperture increased to $5.5 \sigma$, which was comparable to that at the beginning of store. However, from Fig. 13, the actual recovery of $\mathrm{Cu}$ beam lifetime took a much longer time.

Then we calculate the dynamic aperture for the $\mathrm{Cu}$ beam with different ratios of $\mathrm{Cu}$ and $\mathrm{Au}$ beam transverse emittances. In this simulation, we assume that the $\mathrm{Au}$ bunch intensity is $1.3 \times 10^{9}$. We scan the rms transverse emittances of the Au beam from $0.5 \mu \mathrm{m}$ to $1.5 \mu \mathrm{m}$. Three cases are studied: (i) the $\mathrm{Cu}$ beam's transverse emittance always equal to the $\mathrm{Au}$ beam's, (ii) the $\mathrm{Cu}$ beam's transverse emittance always 50\% larger than the $\mathrm{Au}$ beam's, and (iii) the $\mathrm{Cu}$ beam's transverse emittance always double the Au beam's. Figure 15 shows the results from this simulation.

From Fig. 15, the dynamic aperture drops sharply for all three cases when the transverse emittance of the Au beam is below $0.6 \mu \mathrm{m}$. With the transverse emittance of Au beam at $0.5 \mu \mathrm{m}$ and a bunch intensity of $1.3 \times 10^{9}$, the beam-beam parameter for the $\mathrm{Cu}$ beam is 0.023 . The tunes of particles

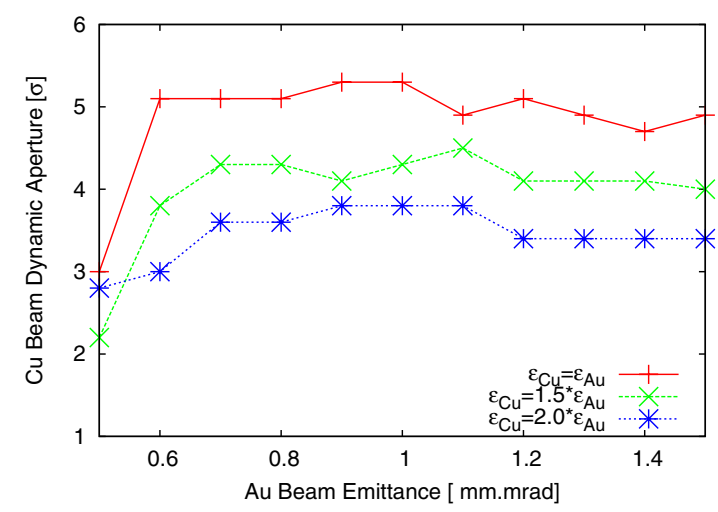

FIG. 15. Calculated dynamic aperture for the $\mathrm{Cu}$ beam with matched and unmatched beam sizes as the $\mathrm{Au}$ beam. The horizontal axis is the Au beam's transverse normalized rms emittance. The Au bunch intensity is $1.3 \times 10^{9}$. 
in the bunch core are pushed onto the 5th order betatron resonance at 0.2 and the dynamic aperture is reduced for all cases.

When the transverse emittance of the Au beam is larger than $0.6 \mu \mathrm{m}$, the emittance matched case gives the highest dynamic aperture around $5 \sigma$. For the cases that the $\mathrm{Cu}$ beam transverse emittance is larger than the Au beam's, the $\mathrm{Cu}$ beam's dynamic aperture is reduced. The larger the difference in the transverse emittances between the $\mathrm{Cu}$ beam and $\mathrm{Au}$ beam, the smaller the $\mathrm{Cu}$ beam dynamic aperture. For the case that the $\mathrm{Cu}$ beam transverse emittance is double that of the $\mathrm{Au}$ beam, the $\mathrm{Cu}$ beam dynamic aperture is only $3.5 \sigma$.

We notice in Fig. 15 that the values of dynamic apertures are not sensitive to the Au beam's emittances when the transverse emittance of the Au beam is larger than $0.6 \mu \mathrm{m}$. This indicates that the dynamic aperture of the $\mathrm{Cu}$ beam is not sensitive to the beam-beam parameter when it is less than 0.019 . The real reason for the large $\mathrm{Cu}$ beam loss is that $\mathrm{Cu}$ ions sample more nonlinearities of the beam-beam interaction force from the smaller sized Au beam.

We also study the effects of unbalanced beam-beam interaction on particles in the satellite buckets. Simulation results show that the dynamic apertures for the $\mathrm{Cu}$ ions in the satellite buckets are also reduced when the $\mathrm{Cu}$ beam emittance is much larger than the Au beam's.

\section{Maximizing integrated luminosity}

The luminosity with different transverse beam sizes is given by
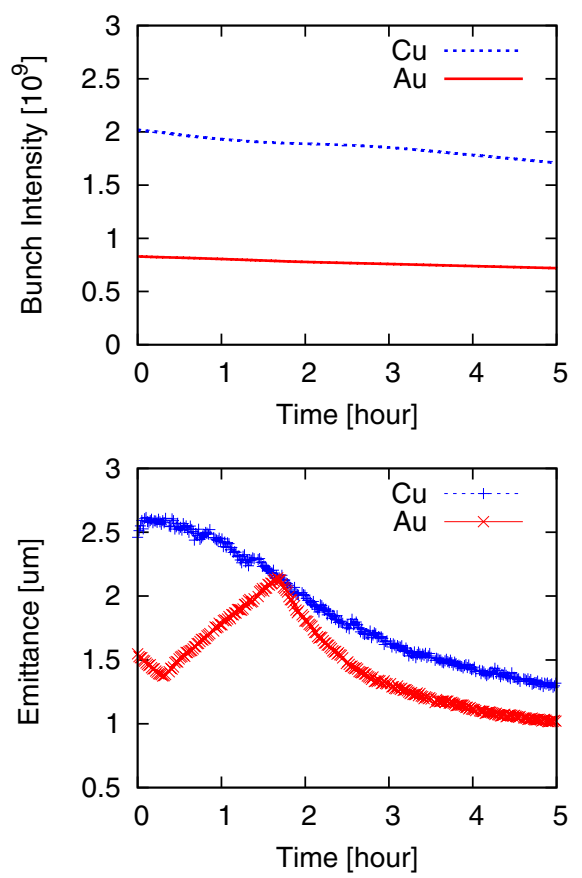

$$
L=\frac{N_{i, 1} N_{i, 2} f_{c}}{2 \pi\left(\sigma_{1}^{* 2}+\sigma_{2}^{* 2}\right)}=\frac{N_{i, 1} N_{i, 2} \gamma f_{c}}{2 \pi \beta^{*}\left(\epsilon_{n, 1}+\epsilon_{n, 2}\right)}
$$

where $f_{c}$ is the frequency of bunch collisions, $\sigma_{1,2}^{*}$ the transverse beam sizes at the IPs, and $\epsilon_{n, 1,2}$ the normalized transverse rms emittances. In Eq. (15), we assumed a round Gaussian particle distribution and same $\beta^{*}$ s for both beams. To achieve a high luminosity, we need to have larger bunch intensities and smaller transverse beam sizes for both beams.

In the $\mathrm{Cu}-\mathrm{Au}$ run, the initial $\mathrm{Cu}$ beam emittance was already 30\% more than the Au beam's. With maximum gains of 3-d cooling for both rings, due to different cooling rates, the difference in the transverse emittances and beam sizes will be further increased. Further reduced Au beam's transverse beam size only slightly increases the instantaneous luminosity. On the other hand, the unbalanced beambeam interaction will cause a lot of $\mathrm{Cu}$ beam loss. For the store shown above in Fig. 13, the Cu beam lost half of its intensity when the $\mathrm{Cu}$ beam's transverse emittance was cooled down, which in return reduced the integrated luminosity for the rest of the store.

To maximize the integrated luminosity, we should reduce the $\mathrm{Cu}$ beam loss at the beginning of store when its transverse emittance was not cooled down. Figure 16 shows the beam parameters of one store during our first attempt. To reduce the difference of transverse emittances between the two beams, here we intentionally turned off the horizontal cooling for the Au beam between 0.3 and 1.7 hours into store. The vertical cooling for the Au beam was
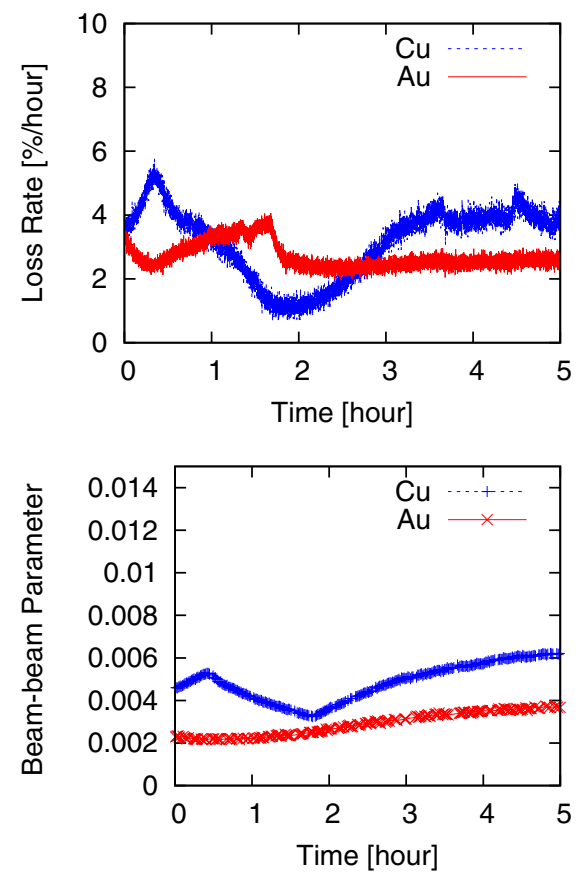

FIG. 16. Beam parameters for the store during our first attempt to slow down Au cooling rate. Top left: bunch intensities. Top right: beam loss rates. Bottom left: normalized transverse emittances. Bottom right: total beam-beam parameters. 


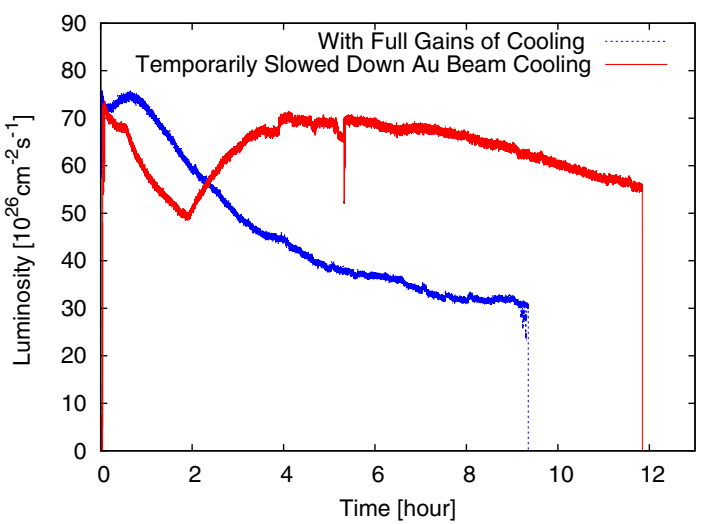

FIG. 17. Comparison of luminosities of the two example fills without and with cooling rate adjustment (as shown in Fig. 13 and Fig. 16). The integrated luminosity per store increased by $74 \%$ with cooling gain adjustment.

kept on to slow down the transverse emittance growth from IBS. For the Cu beam, full gain 3-d cooling was kept on for the entire store.

During this period when the horizontal cooling of $\mathrm{Au}$ beam was shut off, although the instantaneous luminosity decreased, there was just a little intensity loss to the $\mathrm{Cu}$ beam. The difference in the emittances of both beams was continuously reduced. At 1.7 hours into the store, the emittances were comparable and we turned on the horizontal cooling for the Au beam. The luminosity began to increase and reached the initial one at the beginning of store. With full gains of 3-d cooling for both beams in the rest of store, high luminosity lasted for the next several hours. In this store, the beam-beam parameter for the $\mathrm{Cu}$ beam was below 0.006 . The $\mathrm{Cu}$ beam loss rate was below $6 \%$ /hour. Figure 17 compares the luminosities from above two example fills without and with cooling rate adjustment. The integrated luminosity per store increased by $74 \%$ with cooling gain adjustment.

Besides temporarily turning off the horizontal cooling for the $\mathrm{Au}$ beam, we also tested other ways to reduce the $\mathrm{Cu}$ beam loss at the beginning of store. For an example, we could adjust the transverse cooling rate for the Au beam by temporarily switching off some channels of transverse cooling to achieve smoother evolutions of emittances and luminosity. There are 16 channels with different frequencies for each plane of the RHIC transverse cooling system. We also observed that reduced longitudinal cooling rate could slow down the transverse cooling. By slowing down the Au beam's transverse cooling rate at the beginning of store, we reduced the $\mathrm{Cu}$ beam loss at the beginning of store and maximized the integrated luminosity. The $\mathrm{Cu}$ beam loss rate could be controlled below $4 \%$ /hour during the whole store. The store length was extended to 14 hours, which is the longest store length in the RHIC heavy ion runs to date.

\section{CONCLUSIONS}

In this article we analyzed the mechanisms for the large nonluminous beam loss observed in the previous RHIC $100 \mathrm{GeV} \mathrm{Au}-\mathrm{Au}$ runs and conclude that it was mainly caused by a limited off-momentum dynamic aperture. In the 2012 ion run we adopted a new lattice configuration which provides a large off-momentum dynamic aperture and we achieved a burn-off dominated $U$ beam lifetime at physics stores in the $96.4 \mathrm{GeV}$ U-U operation. In the asymmetric $100 \mathrm{GeV} \mathrm{Cu}-\mathrm{Au}$ operation, we observed a large $\mathrm{Cu}$ beam loss at the beginning of store which was caused by the unbalanced beam-beam interaction with different beam sizes at the collisional points stemming from different IBS and cooling rates between the $\mathrm{Au}$ and $\mathrm{Cu}$ ion beams. By intentionally slowing down the cooling rate for the $\mathrm{Au}$ beam, we reduced the $\mathrm{Cu}$ beam loss and maximized the store length and integrated luminosity.

\section{ACKNOWLEDGMENTS}

We would like to thank all our colleagues in the Collider-Accelerator Department at Brookhaven National Laboratory who were involved in the 2012 RHIC U-U and $\mathrm{Cu}-\mathrm{Au}$ runs. For stimulating discussions, we would like to thank M. Bai, A. Drees, A. Fedotov, H. Huang, V. Litvinenko, A. Marusic, C. Montag, M. Minty, S. Tepikian, P. Thieberger, and S. Y. Zhang.

\section{APPENDIX A: BEAM-GAS INTERACTION'S ROLE IN RHIC HEAVY ION RUNS}

The ion beam lifetime and emittance growth due to the interaction between the ions and the residual gas in the RHIC heavy ion operation had been calculated in $[46,47]$. Here we recalculate them with the parameters of current RHIC vacuum system and ion run lattices. In the normal RHIC operation, $83 \%$ of the RHIC beam pipe is at $4.5 \mathrm{~K}$ with a pressure of 0.01 nTorr and the rest at the room temperature $300 \mathrm{~K}$ with a pressure of $0.5 \mathrm{nTorr}$. The warm sections are mainly in the 6 interaction regions. The residual gases are made of $100 \% \mathrm{H}_{\mathrm{e}}$ gas in the cold region and $95 \% \mathrm{H}_{2}$ and $5 \% \mathrm{CO}$ in the warm region. The averaged $\beta$ function is $45 \mathrm{~m}$ in the warm section and $115 \mathrm{~m}$ in the cold section.

Interactions between the stored ions and the residual gas includes inelastic and elastic interactions. For the RHIC ion operation with the particle energy at about $100 \mathrm{GeV} /$ nucleon, the most important interactions are the inelastic nuclear collision and the elastic nuclear Coulomb scattering between the nuclei of ions and gas atoms. The inelastic nuclear collision causes ion loss, while the multiple elastic nuclear Coulomb scattering causes ion beam emittance growth. 


\section{Inelastic nuclear collision}

The cross section for the nuclear collision can be estimated with [48]

$$
\sigma_{N} \simeq \pi R_{N}^{2}
$$

with $R_{N}=r_{0}\left(A_{i}^{1 / 3}+A_{t}^{1 / 3}\right), r_{0} \simeq 1.2 \mathrm{fm}, A_{i, t}$ are the atom mass numbers of the projectile ions and the target gas atoms. The cross sections of nuclear collision between the nuclei of $\mathrm{Au}$ ions and the residual gas atoms are in an order of $10^{-24} \mathrm{~cm}^{-2}$.

For the RHIC $100 \mathrm{GeV} \mathrm{Au-Au} \mathrm{operation,} \mathrm{the} \mathrm{Au} \mathrm{ion}$ beam lifetime due to the nuclear collision is 623 hours in the warm section and 217 hours in the cold region. Overall, the $\mathrm{Au}$ ion beam lifetime due to the inelastic nuclear collision between the nuclei of ions and the gas atoms is 160 hours, or $0.6 \% /$ hour for the Au ion loss rate.

\section{Elastic nuclear collision}

The cross section of single elastic Coulomb scattering between the nuclei of ions and the gas atoms is given by Rutherford cross section. The averaged square of scattering angle $\theta$ is [49]

$$
\left\langle\theta^{2}\right\rangle=2 \theta_{\min }^{2} \ln \left(\frac{\theta_{\max }}{\theta_{\min }}\right)=4 \theta_{\min }^{2} \ln \left(204 Z_{t}^{-1 / 3}\right),
$$

where $\theta_{\min }$ is the minimum scattering angle due to screening of electrons of the target atoms, $\theta_{\max }$ the maximum scattering angle due to finite sizes of target nuclei, $Z_{t}$ the atom number of the target atom. For the RHIC $100 \mathrm{GeV}$ Au-Au operation, $\theta_{\min }$ is in an order of $10^{-10} \mathrm{rad}, \theta_{\max }$ in an order of $10^{-6} \mathrm{rad}$, and $\sqrt{\left\langle\theta^{2}\right\rangle}$ in an order of $10^{-9}$. The rms angle spread $\sigma_{x^{\prime}}$ of the ion beam is in an order of $10^{-5} \mathrm{rad}$. Therefore single elastic Coulomb scattering does not cause ion beam loss.

The growth rate of the rms normalized emittance of the ion beam due to multiple elastic Coulomb scattering between the nuclei of ions and gas atoms is calculated with [50]

$$
\frac{d \epsilon_{n}}{d t}=2 \pi \gamma_{i}\langle\beta\rangle n_{t} \beta_{i} c\left(\frac{2 Z_{i} Z_{t} r_{p}}{A_{i} \beta_{i}^{2} \gamma_{i}}\right)^{2} \ln \left(204 Z_{t}^{-1 / 3}\right),
$$

where $\beta_{i} c$ and $\gamma_{i}$ are the velocity and the relativistic factor of the projectile ions, $n_{t}$ is the particle density of gas atoms. $\langle\beta\rangle$ is the averaged $\beta$ function along the ring.

For the $100 \mathrm{GeV}$ RHIC Au-Au operation, the emittance growth rate is $4.2 \times 10^{-4} \mu \mathrm{m} /$ hour in the warm region and $4.5 \times 10^{-3} \mu \mathrm{m} /$ hour in the cold region. Overall, the emittance growth rate due to multiple elastic Coulomb scattering between the ion nuclei of ions and gas atoms is $4.9 \times 10^{-3} \mu \mathrm{m} /$ hour, or a growth rate of $0.2 \%$ /hour for the normalized emittance. Compared to the IBS effect, the emittance growth from beam-gas interaction can be neglected.

\section{APPENDIX B: TOUSCHEK EFFECT'S ROLE TO MOVE IONS OUT OF THE CENTRAL BUCKET}

For a bunch with a 3-d Gaussian distribution, the beam loss rate due to Touschek effect can be calculated with $[7,51]$

$$
\begin{aligned}
\tau_{T}^{-1}= & \left\langle\frac{r_{i}^{2} c \beta_{x} \beta_{y} \sigma_{h} N_{i}}{8 \sqrt{\pi} \beta^{2} \gamma^{4} \sigma_{x \beta}^{2} \sigma_{y \beta}^{2} \sigma_{s} \sigma_{p}}\right. \\
& \times \int_{u_{m}}^{\infty}\left[\left(2+\frac{1}{u}\right)^{2}\left(\frac{u / u_{m}}{1+u}-1\right)+1\right. \\
& \left.\left.-\frac{\sqrt{1+u}}{\sqrt{u / u_{m}}}-\frac{1}{2 u}\left(4+\frac{1}{u}\right) \ln \frac{u / u_{m}}{1+u}\right)\right] \\
& \left.\times e^{-B_{1} u} I_{0}\left(B_{2} u\right) \frac{\sqrt{u} d u}{\sqrt{1+u}}\right\rangle,
\end{aligned}
$$

with

$$
\begin{gathered}
\frac{1}{\sigma_{h}^{2}}=\frac{1}{\sigma_{p}^{2}}+\frac{D_{x}^{2}+\tilde{D}_{x}^{2}}{\sigma_{x \beta}^{2}}+\frac{D_{y}^{2}+\tilde{D}_{y}^{2}}{\sigma_{y \beta}^{2}}, \\
u_{m}=\beta^{2} \delta_{m}^{2}, \\
\delta_{m}=\Delta p_{m} / p_{0},
\end{gathered}
$$

and

$$
\begin{gathered}
B_{1}=\frac{1}{2 \beta^{2} \gamma^{2}}\left[\frac{\beta_{x}^{2}}{\sigma_{x \beta}^{2}}\left(1-\frac{\sigma_{h}^{2} \tilde{D}_{x}^{2}}{\sigma_{x \beta}^{2}}\right)+\frac{\beta_{y}^{2}}{\sigma_{y \beta}^{2}}\left(1-\frac{\sigma_{h}^{2} \tilde{D}_{y}^{2}}{\sigma_{y \beta}^{2}}\right)\right], \\
B_{2}=\sqrt{B_{1}^{2}-\frac{\beta_{x}^{2} \beta_{y}^{2} \sigma_{h}^{2}}{\beta^{4} \gamma^{4} \sigma_{x \beta}^{2} \sigma_{y \beta}^{2}}\left(\frac{1}{\sigma_{p}^{2}}+\frac{D_{x}^{2}}{\sigma_{x \beta}^{2}}+\frac{D_{y}^{2}}{\sigma_{y \beta}^{2}}\right)}
\end{gathered}
$$

Here $I_{0}$ is the modified Bessel function, $\tilde{D}_{x, y}=D_{x, y}^{\prime} \beta_{x, y}-$ $D_{x, y} \beta_{x, y}^{\prime} / 2, \sigma_{x \beta, y \beta}$ are the horizontal and vertical rms betatron beam widths, $\Delta p_{m}$ the maximum stable momentum deviation.

Equation (B1) assumes that the momentum spread of particles is much smaller than the maximum momentum acceptance $\delta_{m}$. For the RHIC ion run, particles already fill up the central $197 \mathrm{MHz}$ RF bucket. Particles do not need to have a relative momentum change larger than the central bucket's momentum acceptance $1.4 \times 10^{-3}$ to get out of it. However, we still can use Eq. (B1) to calculate the possibility of particles with a relative momentum change $\delta_{m}$. Figure 18 shows the percentage of Au ions per hour having a relative momentum change $\delta_{m}>0, \delta_{m}$ is scanned from $5 \times 10^{-5}$ to $1.4 \times 10^{-3}$ with a step size of $5 \times 10^{-5}$.

For a particle with relative momentum deviation $\delta>0$, it will be moved out of the central bucket if its relative momentum change due to Touschek effect is larger than 


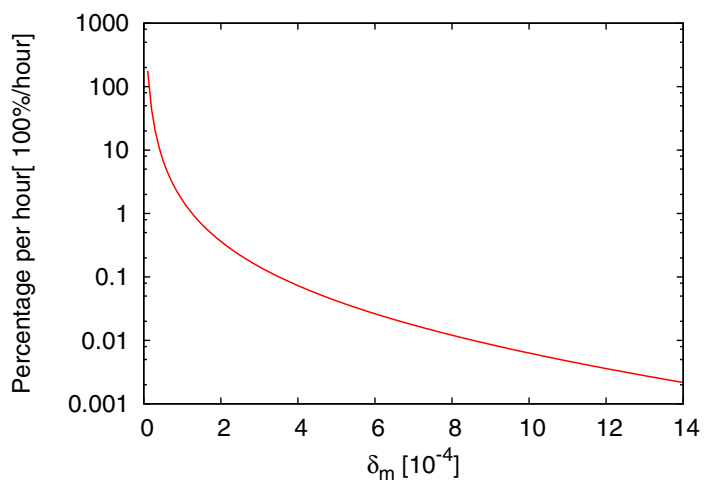

FIG. 18. Percentage of $\mathrm{Au}$ ions per hour having a relative momentum change $\delta_{m}$ due to the Touschek effect. In this calculation, a 3-d Gaussian distribution bunch is assumed.

$\left(1.4 \times 10^{-3}-\delta\right)$. For a bunch with a Gaussian distribution, the particle leakage rate from the central bucket due to Touschek effect is

$$
\tau_{\text {leakage }}^{-1}=\int_{0}^{1.4 \times 10^{-3}} \tau_{T}^{-1}\left(1.4 \times 10^{-3}-\delta\right) \rho(\delta) d \delta .
$$

Here $\tau_{T}^{-1}\left(\delta_{m}\right)$ is the loss rate due to Touschek effect with a maximum momentum acceptance $\delta_{m} . \rho(\delta)$ is the 1-D Gaussian distribution of relative momentum deviation. The contribution from the particles with negative $\delta$ s is already included in Eq. (B7).

We assume the bunch's rms relative momentum spread to be $1 / 3$ of the momentum acceptance of central bucket. By numerically integrating Eq. (B7), we obtain the total leakage rate due to Touschek effect about $3.7 \%$ /hour of the total beam intensity at the beginning of store in the $100 \mathrm{GeV} \mathrm{Au}-\mathrm{Au}$ operation. Considering a total leakage rate of about $20 \%$ /hour out of the central bucket, Touschek effect's contribution is smaller than IBS. After 2.5 hours into store, the $\mathrm{Au}$ beam was cooled down, the particle leakage rate due to Touschek effect increased to 5.4\%/hour. With a total leakage rate of $6.5 \%$ /hour, Touschek effect became the main source to move particles out of the central RF bucket.

For the $96.4 \mathrm{GeV}$ U-U operation in 2012, at the beginning of store, with a bunch intensity $0.3 \times 10^{9}$ and a transverse rms emittance $1.6 \mu \mathrm{m}$, the leakage rate from the central RF bucket due to Touschek effect is $1.4 \%$ /hour. With a total leakage rate of $10 \%$ /hour, IBS is the main reason to move particles out of the central bucket. After the $\mathrm{U}$ beam was cooled down at 1 hour into store, with a bunch intensity $0.24 \times 10^{9}$ and a transverse rms emittance $0.33 \mu \mathrm{m}$, Touschek effect generates a leakage rate of $10.2 \%$ /hour and plays a much bigger role than IBS to move particles out of the central bucket. After 3 hours into store, the total particle leakage rate from the central buckets decreases to $4 \%$ /hour.
[1] W. Fischer, in Proceedings of the International Particle Accelerator Conference, Kyoto, Japan (ICR, Kyoto, 2010), p. 1227.

[2] W. Fischer, RHIC run overview, http://www.agshome.bnl .gov/RHIC/Runs/.

[3] J. G. Alessi, D. Barton, E. Beebe, S. Bellavia, O. Gould, A. Kponou, R. Lambiase, R. Lockey, A. McNerney, M. Mapes, Y. Marneris, M. Okamura, D. Phillips, A. I. Pikin, D. Raparia, J. Ritter, L. Snydstrup, C. Theisen, and M. Wilinski, Rev. Sci. Instrum. 81, 02 A509 (2010).

[4] J. G. Alessi, E. Beebe, S. Binello, L. Hoff, K. Kondo, R. Lambiase, V. LoDestro, M. Mapes, A. McNerney, J. Morris, M. Okamura, A. I. Pikin, D. Raparia, J. Ritter, L. Smart, L. Snydstrup, M. Wilinski, and A. Zaltsman, in Proceedings of the 25th International Linear Accelerator Conference, LINAC-2010, Tsukuba, Japan (KEK, Tsukuba, Japan, 2010), p. 1033.

[5] A. Pikin, J. G. Alessi, E. N. Beebe, A. Kponou, R. Lambiase, R. Lockey, D. Raparia, J. Ritter, L. Snydstrup, and Y. Tan, JINST 5, C09003 (2010).

[6] P. Thieberger, Nucl. Instrum. Methods 220, 45 (1984).

[7] V. Lebedev, in Handbook of Accelerator Physics and Engineering, edited by A. Chao, K. H. Mess, M. Tigner, and F. Zimmermann (World Scientific, Singapore, 2013).

[8] A. Piwinski, in Frontiers of Particle Beams, edited by M. Month and S. Turner (Springer, New York, 1988), p. 297.

[9] J. Bjorken and S. Mtingwa, Part. Accel. 13, 115 (1983).

[10] M. Martini, Part. Accel. 17, 1 (1985).

[11] V. Lebedev, in 33rd ICFA Advanced Beam Dynamics Workshop on High Intensity and High Brightness Hadron Beams, AIP Conf. Proc. No. 773, edited by I. Hoffman, J. M. Lagniel, and R. W. Hasse (AIP, New York, 2005), p. 440.

[12] W. Fischer, M. Bai, M. Blaskiewicz, J. M. Brennan, P. Cameron, R. Connolly, A. Lehrach, G. Parzen, S. Tepikian, $\mathrm{K}$. Zeno, and J. van Zeijts, in Proceedings of the Particle Accelerator Conference, Chicago, IL, 2001 (IEEE, New York, 2001), p. 2857.

[13] A. V. Fedotov, W. Fischer, S. Tepikian, and J. Wei, in Proceedings of ICFA Hadron Beams 2006, Tsukuba, Japan, 2006, p. 259, http://accelconf.web.cern.ch/ AccelConf/abdwhb06/PAPERS/WEB Y03.PDF.

[14] V. N. Litvinenko et al., in Proceedings of the 11th European Particle Accelerator Conference, Genoa, 2008 (EPS-AG, Genoa, Italy, 2008), p. 2557.

[15] A. V. Fedotov et al., in Proceedings of Hadron Beam 2008, Nashville, Tennessee, USA, p. 148-152, http://accelconf .web.cern.ch/AccelConf/HB2008/papers/wga28.pdf.

[16] M. Blaskiewicz, in Handbook of Accelerator Physics and Engineering, edited by A. Chao, K. H. Mess, M. Tigner, and F. Zimmermann (World Scientific, Singapore, 2013).

[17] S. van der Meer, Report No. CERN/ISR-PO/72-31, 1972; Rev. Mod. Phys. 57, 689 (1985).

[18] M. Blaskiewicz and J. M. Brennan, Phys. Rev. ST Accel. Beams 10, 061001, 2007.

[19] M. Blaskiewicz, J. M. Brennan, and F. Severino, Phys. Rev. Lett. 100, 174802 (2008).

[20] M. Blaskiewicz, J. M. Brennan, and K. Mernick, Phys. Rev. Lett. 105, 094801 (2010). 
[21] A. Drees et al., in Proceedings of the 22nd Particle Accelerator Conference, PAC-2007, Albuquerque, NM (IEEE, New York, 2007), p. 722.

[22] C. J. Gardner et al., in Proceedings of the 11th European Particle Accelerator Conference, Genoa, 2008 (EPS-AG, Genoa, Italy, 2008), p. 2548.

[23] K. A. Brown et al., in Proceedings of the International Particle Accelerator Conference, Kyoto, Japan (ICR, Kyoto, 2010), p. 507.

[24] G. J. Marr et al., in Proceedings of the 2nd International Particle Accelerator Conference, San Sebastián, Spain (EPS-AG, Spain, 2011), p. 1894.

[25] Y. Luo et al., in Proceedings of the 4th International Particle Accelerator Conference, IPAC-2013, Shanghai, China, 2013 (JACoW, Shanghai, China, 2013), p. 1538.

[26] W. Fischer, J. M. Brennan, M. Blaskiewicz, and T. Satogata, Phys. Rev. ST Accel. Beams 5, 124401 (2002).

[27] W. Fischer, M. Blaskiewicz, J. M. Brennan, H. Huang, H.-C. Hseuh, V. Ptitsyn, T. Roser, P. Thieberger, D. Trbojevic, J. Wei, S. Y. Zhang, and U. Iriso, Phys. Rev. ST Accel. Beams 11, 041002 (2008).

[28] S. Y. Zhang, H.C. Hseuh, P. Thieberger, and D. Trbojevic, Phys. Rev. ST Accel. Beams 8, 123201 (2005).

[29] S. Y. Zhang and V. Ptitsyn, Phys. Rev. ST Accel. Beams 11, 051001 (2008).

[30] Y. Luo, K. Brown, W. Fischer, G. Marr, G. RobertDemolaize, T. Roser, V. Schoefer, S. Tepikian, and D. Trbojevic, in Proceedings of the 2nd International Particle Accelerator Conference, San Sebastián, Spain (EPS-AG, Spain, 2011), p. 1891.

[31] Y. Luo, M. Bai, M. Blaskiewicz, W. Fischer, X. Gu, A. Marusic, T. Roser, S. Tepikian, and S. Zhang, in Proceedings of the 3rd International Particle Accelerator Conference, New Orleans, LA, 2012 (IEEE, Piscataway, NJ, 2012), p. 175.

[32] W. Fischer, A. J. Baltz, M. Blaskiewicz, D. Gassner, K. A. Drees, Y. Luo, M. Minty, P. Thieberger, and M. Wilinski, Phys. Rev. C 89, 014906 (2014).

[33] M. Blaskiewicz, in Proceedings of COOL 2007, Bad Kreuznach, Germany, p. 125, http://accelconf.web.cern .ch/AccelConf/c107/PAPERS/WEM2I05.PDF.
[34] Y. Luo, in Proceedings of the International Particle Accelerator Conference, Kyoto, Japan (ICR, Kyoto, 2010), p. 1907.

[35] S. Tepikian, W. Fischer, Y. Luo, and V. Ptitsyn, in 2006 RHIC Retreat Workshop, 2006.

[36] P. J. Bryant, CERN Report No. 95-06, edited by S. Turner, 1995.

[37] S. Y. Lee, Accelerator Physics (World Scientific, Singapore, 1999).

[38] Y. Luo, M. Bai, J. Beebe-Wang, J. Bengtsson, R. Calaga, W. Fischer, A. Jain, N. Malitsky, S. Peggs, F. Pilat, V. Ptitsyn, G. Robert-Demolaize, T. Satogata, S. Tepikian, and D. Trbojevic, in Proceedings of the 23rd Particle Accelerator Conference, Vancouver, Canada, 2009 (IEEE, Piscataway, NJ, 2009), p. 2489.

[39] Y. Luo, W. Fischer, S. Tepikian, and D. Trobjevic, in Proceedings of the 22nd Particle Accelerator Conference, PAC-2007, Albuquerque, NM (IEEE, New York, 2007), p. 4357.

[40] Y. Luo, S. Tepikian, W. Fischer, G. Robert-Demolaize, and D. Trbojevic, Brookhaven National Laboratory (BNL) C-AD AP-Note 348, 2009.

[41] MAD-Methodical Accelerator Design, CERN. http://mad .web.cern.ch $/ \mathrm{mad} /$.

[42] M. Borland, Elegant: A Flexible SDDS-Compliant Code for Accelerator Simulation, Advanced Photon Source LS-287, 2000.

[43] A. Pshenichnov (private communication).

[44] K. Hirata, in Handbook of Accelerator Physics and Engineering, edited by A. Chao, K. H. Mess, M. Tigner, and F. Zimmermann (World Scientific, Singapore, 2013).

[45] K. Hirata, H. Moshammer, and F. Ruggiero, Part. Accel. 40, 205 (1993).

[46] M. J. Rhoades-Brown and M. Harrison, BNL Report No. 47070, 1991 (unpublished).

[47] D. Trbojevic, BNL RHIC AP Report No. 136, 1997 (unpublished).

[48] L. D. Landau and E. M. Lifshitz, Mechanics (Pergamon Press, Oxford, 1960).

[49] J. D. Jackson, Classic Electrodynamics (John Wiley \& Sons, New York, 1975).

[50] B. Frankzke, CERN Yellow Report No. 92-01, 1992, pp. 100-120.

[51] A. Piwinsk, DESY Report No. 98-179 (unpublished). 